\title{
THE COUNTING VECTOR OF A SIMPLE GAME ${ }^{1}$
}

\author{
EITAN LAPIDOT ${ }^{2}$
}

\begin{abstract}
The counting vector of a simple game is the vector $f=(f(1), f(2), \cdots, f(n))$ where $f(i)$ is the number of winning coalitions containing the player $i$. In this paper, we show that the counting vector of a weighted majority game determines the game uniquely. With the aid of the counting vector we find an upper bound on the number of weighted majority games.
\end{abstract}

1. Preliminaries on simple games. A simple game is a pair $G=$ $(N ; W)$, where $N=\{1,2, \cdots, n\}$ is a set of $n$ members and $W$ is a set of subsets of $N$. The members of $N$ are called players; subsets of $N$ are called coalitions. The elements of $W$ are called winning coalitions. A simple game is called monotone if every superset of a winning coalition is itself a winning coalition. A weighted majority game is a simple game for which there exist $n$ nonnegative numbers $w_{1}, w_{2}, \cdots, w_{n}$ and a positive number $q$, such that, $S$ is a winning coalition if and only if $w(S)=\sum_{i \in S} w_{i} \geqq q . \quad w=\left[w_{1}, w_{2}, \cdots, w_{n} ; q\right]$ is called the representation of the game. A weighted majority game is denoted by $G=(N ; w)$ where $w$ is its representation.

$G$ is called constant-sum if for each coalition $S$ exactly one of the two coalitions $S$ and $N-S$ is winning.

2. The counting vector theorem. Given a simple game $G=(N ; W)$, we denote by $f(i)$ the number of winning coalitions containing player $i$, and by $f$ the vector $f=(f(1), f(2), \cdots, f(n))$ called the counting vector of the game.

\section{THEOREM 2.1. Let}

and

$$
G_{1}=\left(N ;\left[w^{(1)}, w_{2}^{(1)}, \cdots, w_{n}^{(1)} ; q^{(1)}\right]\right)
$$

$$
G_{2}=\left(N ;\left[w_{1}^{(2)}, w_{2}^{(2)}, \cdots, w_{n}^{(2)} ; q^{(2)}\right]\right)
$$

Received by the editors November 23. 1970.

AMS 1970 subject classifications. Primary 90D12.

Key words and phrases. Simple games, weighted majority game, counting vector, desirability relation, symmetric players.

${ }^{1}$ This paper is contained in an M.Sc. thesis submitted to the Technion, Israel Institute of Technology in 1968.

${ }^{2}$ The author wishes to express his deep gratitude to Dr. B. Peleg for his guidance and inspiration. 
be two weighted majority games. Their counting vectors $f_{1}$ and $f_{2}$ are equal if and only if $G_{1}=G_{2}$.

Proof. Suppose that $G_{1} \neq G_{2}$ but $f_{1}=f_{2}$, i.e., for every $i \in N$,

$$
f_{1}(i)=f_{2}(i) \text {. }
$$

(We may assume that $f \neq 0$ since otherwise we have the trivial game $(N ; \phi)$.) Let $W_{1}, W_{2}$ be the sets of winning coalitions in $G_{1}, G_{2}$ respectively. We set $\Omega_{1}=W_{1}-W_{2}, \Omega_{2}=W_{2}-W_{1}$. If $W_{k} \subset W_{p}(k ; p=1,2$, $k \neq p)$, then $f_{k}(i) \leqq f_{p}(i)$ for every $i$ and inequality holds for at least one player $i_{0}$, in contradiction to (2.1); hence,

$$
\Omega_{1} \neq \phi, \quad \Omega_{2} \neq \phi .
$$

For each $S \in \Omega_{1}$ and $T \in \Omega_{2}$ we have

$$
w^{(1)}(S)>w^{(1)}(T) .
$$

Summing these inequalities over $\Omega_{2}$ for a fixed $S \in \Omega_{1}$, we have

$$
\left|\Omega_{2}\right| w^{(1)}(S)>\sum_{T \in \Omega_{2}} w^{(1)}(T)
$$

where $|\Omega|$ is the number of elements of $\Omega$. Summing all the inequalities (2.4) over $\Omega_{1}$, we have

$$
\left|\Omega_{2}\right| \sum_{S \in \Omega_{1}} w^{(1)}(S)>\left|\Omega_{1}\right| \sum_{T \in \Omega_{2}} w^{(1)}(T) .
$$

Let $\phi_{k}(i)$ be the number of coalitions in $\Omega_{k}$ containing player $i$. Since $\Omega_{1}=W_{1}-\left(W_{1} \cap W_{2}\right)$ and $\Omega_{2}=W_{2}-\left(W_{1} \cap W_{2}\right), f_{1}(i)=f_{2}(i)$ implies $\phi_{1}(i)=\phi_{2}(i)$. Denoting the common value by $\phi(i)$, we have

$$
\begin{aligned}
& \sum_{S \in \Omega_{1}} w^{(1)}(S)=\sum_{i \in N} \phi(i) w_{i}^{(1)}, \\
& \sum_{T \in \Omega_{2}} w^{(1)}(T)=\sum_{i \in N} \phi(i) w_{i}^{(1)} .
\end{aligned}
$$

Since $q^{(1)}$ is positive and $\Omega_{1} \neq \phi, \Omega_{2} \neq \phi$, we have, from (2.5), (2.6), and (2.7),

$$
\left|\Omega_{1}\right|<\left|\Omega_{2}\right| \text {. }
$$

Using the representation $\left[w_{1}^{(2)}, w_{2}^{(2)}, \cdots, w_{n}^{(2)} ; q^{(2)}\right]$ of $G_{2}$ we have

$$
\left|\Omega_{2}\right|<\left|\Omega_{1}\right|
$$

in contradiction to $(2.8)$ and hence $f_{1} \neq f_{2}$. 
Corollary 2.1. Let $G_{1}=\left(N ; W_{1}\right)$ be a weighted majority game and $G_{2}=\left(N ; W_{2}\right)$ a simple game. If $G_{1} \neq G_{2}$ and

$$
\left|W_{1}\right| \geqq\left|W_{2}\right| \text {, }
$$

then $f_{1} \neq f_{2}$.

Proof. (2.10) implies

$$
\left|\Omega_{1}\right| \geqq\left|\Omega_{2}\right| \text {. }
$$

If $f_{1}=f_{2}$ then as in the proof of Theorem 2.1, $\left|\Omega_{1}\right|<\left|\Omega_{2}\right|$. Hence $f_{1} \neq f_{2}$.

COROLlARY 2.2. Let $G_{1}$ be a constant-sum weighted majority game and $G_{2}$ a constant-sum simple game. If $G_{1} \neq G_{2}$, then $f_{1} \neq f_{2}$.

Proof. In any constant-sum simple game, the number of winning coalitions is $2^{n-1}$ ( $n$ being the number of players), hence $\left|W_{1}\right|=\left|W_{2}\right|$. The assumptions of Corollary 2.1 are valid, hence $f_{1} \neq f_{2}$.

3. An upper bound on the number of weighted majority games. In [1], J. R. Isbell raised the problem of finding an upper bound on the number of weighted majority games. No answer was given, except for the bound $2^{2^{n}}$, which is the number of sets of coalitions in $N$. Using the counting vector concept a smaller value is obtainable for the upper bound.

It is evident that for every $i, 0 \leqq f(i) \leqq 2^{n-1}$. If $f(i)=0$ for some $i$, then $N \notin W$; hence $W=\phi$, i.e., the game is the trivial game $G_{0}=(N ; \phi)$. For all other games $1 \leqq f(i) \leqq 2^{n-1}$. The number of vectors

$$
a=\left(a_{1}, a_{2}, \cdots, a_{n}\right), \quad a_{i}=1,2, \cdots, 2^{n-1}, \quad i=1,2, \cdots, n,
$$

is $2^{n(n-1)}$. Since not every vector of the type (3.1) is a counting vector, there are less than $2^{n(n-1)}$ weighted majority $n$-person games.

Two games, $G_{1}=\left(N ; W_{1}\right)$ and $G_{2}=\left(N ; W_{2}\right)$, are said to be equivalent if there exists a permutation $\pi$ of $N$ such that

$$
W_{2}=\left\{\pi S: S \in W_{1}\right\}
$$

where $\pi S=\{\pi(i): i \in S\}$.

It is evident that this relation is an equivalence. If we identify equivalent games, $f$ is no longer a vector but an unordered set of $n$ numbers. Hence their number is less than $\left({ }^{2 n-1+n-1}\right)$, the number of $n$-selections of $2^{n-1}$ given elements.

The following example shows that there exist two nonequivalent monotone games which have the same counting vector.

EXAMPLE. Let $N$ be a set of seven players $\{1,2,3,4,5,6,7\} . W_{1}$ consists of all 4, 5, 6, and 7 player coalitions and of the following 3 players coalitions: $(2,5,3),(3,7,1),(1,4,2),(2,6,7),(3,6,4),(1,6,5)$, and 
$(4,5,7) . W_{2}$ consists of all $4,5,6$, and 7 player coalitions and of the following 3 player ones: $(2,4,3),(3,7,1),(1,5,2),(2,6,7),(3,6,4)$, $(1,6,5)$, and $(4,5,7)$.

The two games $\left(N ; W_{1}\right)$ and $\left(N ; W_{2}\right)$ are monotone and it is easy to see that they have the same counting vector. However, $W_{1}$ contains no two disjoint 3 player coalitions while $W_{2}$ does, and thus they are not equivalent.

4. The desirability relation. Two players $i$ and $j$, in a simple game $(N ; W)$ are called symmetric if for every coalition $S$ not containing $i$ nor $j$, $S \cup\{i\}$ is winning if and only if $S \cup\{j\}$ is winning; this is denoted by $i \sim j$. If for every coalition $S$ not containing $i$ nor $j, S \cup\{j\} \in W$ implies $S \cup\{i\} \in W$, then $i$ is said to be more desirable than $j$; this is denoted by $i \succsim j$ :

If $i \succsim j$ and $j \succsim i$ then $i \sim j$. If $i \succsim j$ but $i$ and $j$ are not symmetric, we say that $i$ is strictly more desirable than $j$; this is denoted by $i>j$. In a weighted majority game with a representation $\left[w_{1}, w_{2}, \cdots, w_{n} ; q\right]$, $w_{1} \geqq w_{j}$ implies $i \succsim j$ and there exists a representation $\left[w_{1}^{*}, w_{2}^{*}, \cdots, w_{n}^{*}\right.$; $\left.q^{*}\right]$ such that $i \sim j$ implies $w_{i}^{*}=w^{*}[3]$. The counting vector strictly preserves the desirability relation, i.e., $f(i)>f(j)$ implies $i>j$ and $f(i)=f(j)$ implies $i \sim j$ [2]. This enables us to reduce the number of unknowns when seeking a representation of a given weighted majority game.

\section{REFERENCES}

1. J. R. Isbell, On the enumeration of majority games, Math. Tables Aids Comput. 13 (1959), 21-28. MR 21 \#1912.

2. E. Lapidot, On symmetry-groups of games, development in operations research, Proc. Third Annual Israel Conf. on Operations Research (1969), Gordon and Breach, London, 1970, pp. 571-583.

3. M. Maschler and B. Peleg, A characterization, existence proof and dimension bounds for the kernel of a game, Pacific J. Math. 18 (1966), 289-328. MR 34 \#5525.

Department of Mathematics, Technion, Israel Institute of Technology, HAIFA, ISRAEL 